\title{
Cardiovascular complications induced pheochromocytoma associated with neurofibromatosis type 1 (von Recklinghausen's disease) - case report and review of literature
}

\author{
Ovidiu Mitu, ${ }^{1, *}$, Dan Tesloianu ${ }^{1}$, Ionut Tudorancea ${ }^{1,2}$, Nicolae Danila ${ }^{1,2}$, Antoniu \\ Octavian Petriş ${ }^{1,2}$
}

${ }^{1}$ Cardiology Department, "Sf. Spiridon" Clinical Emergency Hospital, lasi, Romania, ${ }^{2}$ "Grigore T. Popa" University of Medicine and Pharmacy, lasi, Romania

\begin{abstract}
Neurofibromatosis type 1 (also known as von Recklinghausen's disease) is a genetic disorder characterized by neurofibromas, skin pigmentation, eye and bone abnormalities. It is associated with pheochromocytoma in 1 to $5 \%$ of cases which determines symptoms related to catecholamine excessive secretion. We present the case of a 54-year-old man that was admitted to the cardiology clinic due to acute onset of tachyarrhythmia (atrial flutter) and high blood pressure. Neurofibromatosis type 1 was diagnosed by clinical examination while laboratory exams, 24-hour continuous blood pressure monitoring and imaging exams raised the probability of pheochromocytoma. After proper pharmacological stabilization, the patient underwent surgery and the $110 / 110 \mathrm{~mm}$ left adrenal mass was removed via abdominal laparotomy. The patient evolution was favorable and regular follow-up is scheduled. In conclusion, even though asymptomatic screening for pheochromocytoma is not recommended, this pathology should be suspected in all patients with neurofibromatosis especially if they present acute onset of cardiovascular signs.
\end{abstract}

Keywords: neurofibromatosis type 1, von Recklinghausen's disease, pheochromocytoma, cardiovascular, arterial hypertension

\section{Introduction}

Pheochromocytoma (PHEO), although rare, is a tumor of the chromaffin tissue with $90 \%$ localization in the adrenal medulla. It causes an excessive secretion of catecholamines that leads to symptoms such as headaches, excessive sweating, flushing or anxiety as well as cardiovascular signs palpitations, tachycardia or paroxysmal arterial hypertension [1].

Received: October 2014; Accepted after review: November 2014; Published: December 2014

${ }^{*}$ Corresponding author: Ovidiu Mitu, MD, PhD, Cardiology Department, "Sf. Spiridon" Clinical Emergency Hospital, 1 Independentei Boulevard, lasi, Romania. Email: mituovidiu@yahoo.co.uk
PHEO represents one the main causes of secondary arterial hypertension and may be so severe to induce hypertensive emergencies [2]. The diagnosis is important since, in most cases, hypertension is reversible after surgical excision. Most of $\mathrm{PHEO}$ are sporadic but in different genetic disorders, PHEO is associated mainly with multiple endocrine neoplasia type 2, von Hippel-Lindau disease or neurofibromatosis type 1 (NF-1) [3].

NF-1, also known as von Recklinghausen's disease, is an autosomal dominant genetic disease with an incidence of about 1 per 3500 births.

$\mathrm{NF}-1$ is characterized by cutaneous and plexiform neurofibromas, skin pigmentation (axillary and inguinal freckling, café-au-lait 
spots), Lisch nodules, optic glioma or skeletal dysplasias $[4,5]$.

Cardiovascular effects are relatively common in patients with NF-1 due to coartation of the aorta, renal artery stenosis or PHEO [6]. Even though PHEO has tenfold higher incidence in NF-1 patients, this association is identified in 1 up to 5 percent of individuals [7]. Thus, we present the case of a patient that was diagnosed with NF-1 and $\mathrm{PHEO}$ due to initial cardiovascular events and we make a brief review of the literature concerning this rare disease association.

\section{Case report}

We present the case of a 54-year-old man who was admitted in emergency to the Cardiology Clinic of "Sf. Spiridon" Clinical
Emergency Hospital lasi accusing palpitation, dizziness and high blood pressure (BP) values (maximum systolic BP $200 \mathrm{mmHg}$ ).

The patient had no personal history of cardiovascular diseases (CVD) and his symptoms presented an acute onset a couple of hours before presenting to the emergency unit. He was non-smoker, without family history of CVD and he was under chronic medication for an organic depressive disorder.

Clinical evaluation showed a normal weight patient (body mass index $21 \mathrm{~kg} / \mathrm{m}^{2}$ ), resting $\mathrm{BP} 120 / 80 \mathrm{mmHg}$ and heart rate 150 bpm.

The skin examination revealed multiple papilloma lesions of 0.5 to 5 centimeters in diameter located on the anterior and posterior thorax, abdomen and upper limbs that were interpreted as neurofibromas, along with caféau-lait spots and axillary freckling (Figure 1).

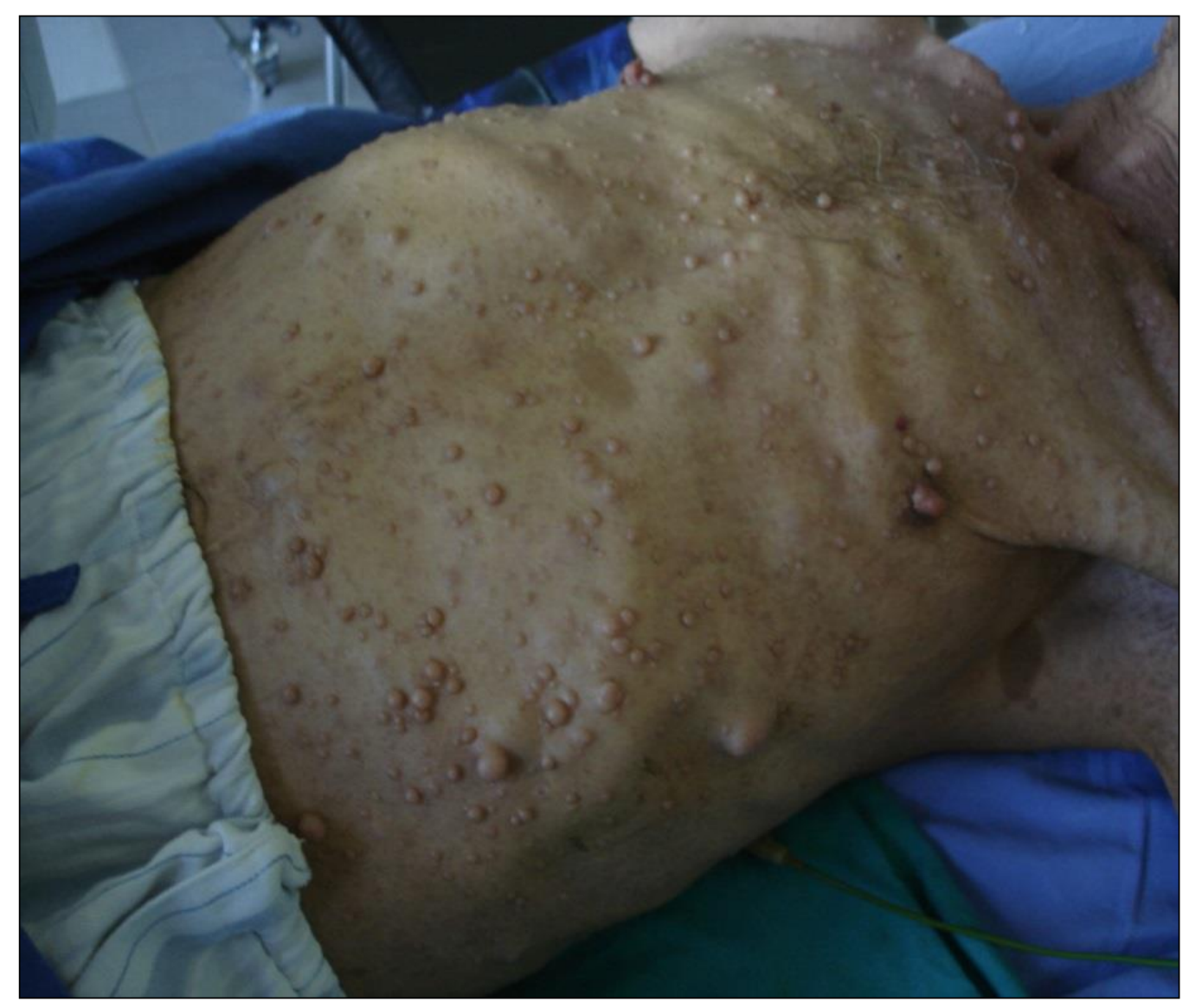

Fig. 1. Axillary freckling plus neurofibromas and café-au-lait spots located on anterior thorax and abdomen

Electrocardiogram at rest revealed atrial flutter with a fast ventricular rate $150 \mathrm{bpm}$ (Figure 2). The 2D transthoracic Doppler echocardiography showed cavities and ventricular walls of normal kinetic and dimensions with an ejection fraction of $50 \%$. 


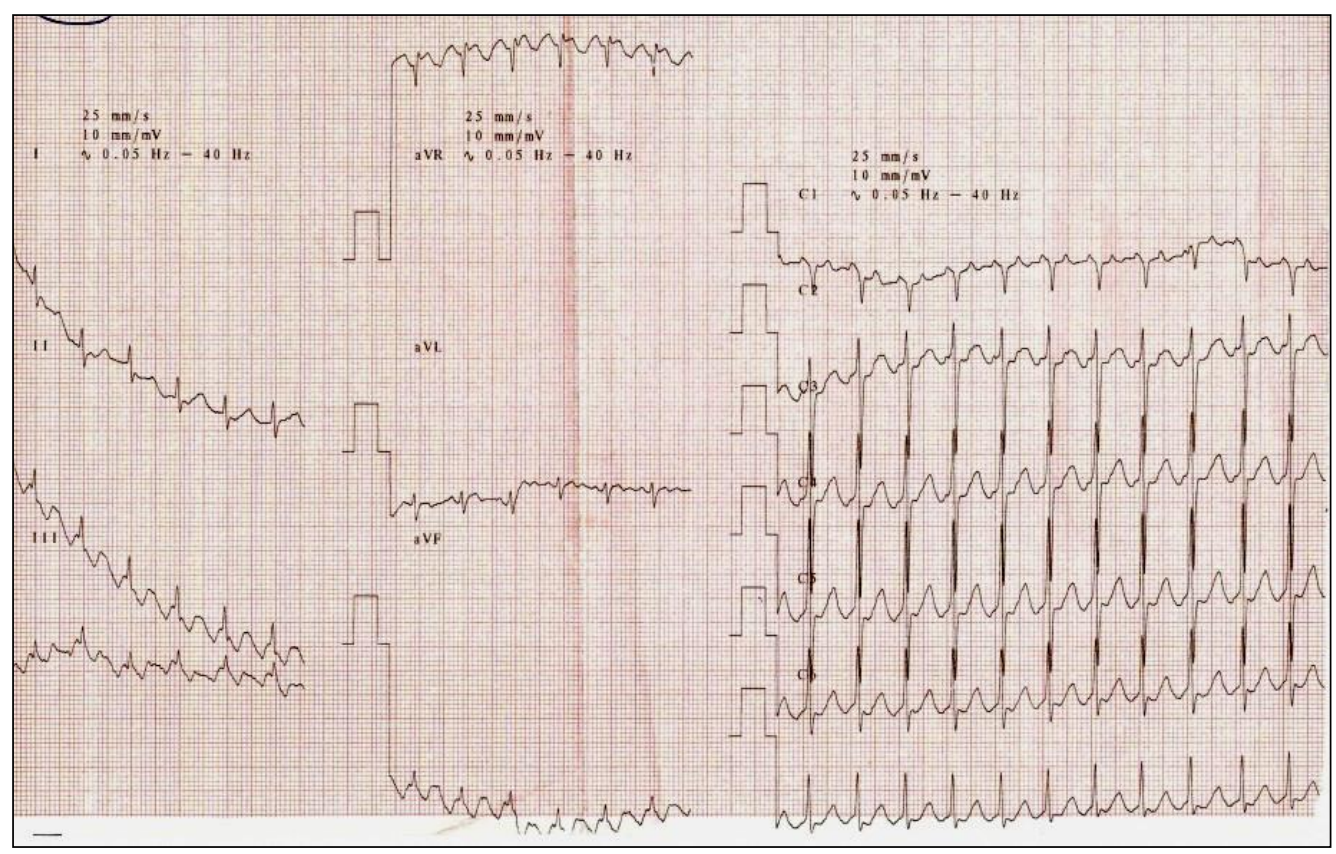

Fig. 2. Atrial flutter with fast ventricular rate $150 \mathrm{bpm}$

Laboratory exams showed multiple abnormal values: mild inflammatory syndrome (increased leukocytes number and fibrinogen) with normal red blood cell count, mild increase of serum creatin kinase (CK) (total CK $705 \mathrm{U} / \mathrm{l}$ and CK-MB $52 \mathrm{U} / \mathrm{I}$ ) and hyperglycemia (175 $\mathrm{mg} / \mathrm{dl})$.

The hepatic and renal functions were impaired as well (AST $68 \mathrm{U} / \mathrm{l}$, ALT $79 \mathrm{U} / \mathrm{l}$, respectively serum creatinine $1.31 \mathrm{mg} / \mathrm{dl}$ ), cholesterol parameters were increased (total cholesterol $242 \mathrm{mg} / \mathrm{dl}$, LDL cholesterol 151 $\mathrm{mg} / \mathrm{dl}$ ) while sodium and potassium levels were in normal ranges.

The 24-hour continuous blood pressure monitoring revealed a disturbed BP profile with important differences between the lowest and highest values (Table 1).

Table 1. 24-hour continuous blood pressure monitoring results

\begin{tabular}{lcc}
\hline & Systolic BP $(\mathrm{mmHg})$ & Diastolic BP $(\mathrm{mmHg})$ \\
\hline Average & 116 & 70 \\
Maximum & 195 & 113 \\
Minimum & 80 & 31 \\
\hline
\end{tabular}

Taking into consideration the clinical presentation and examination, the abnormal laboratory results and the extreme oscillating values obtained by BP monitoring, we thought of a secondary neuro-endocrine cause for the patient cardiovascular manifestations. The abdominal echography revealed an extensive mass of $110 / 114 \mathrm{~mm}$ with a $70 \mathrm{~mm}$ inside necrosis area that was located in the left adrenal gland. The serum catecholamine levels were elevated (metanephrine $420 \mathrm{pg} / \mathrm{ml}$ and normetanephrine $1000 \mathrm{pg} / \mathrm{ml}$ ). Thus, based on clinical symptoms, echography and biochemical markers the diagnosis of pheochromocytoma was likely probable. The abdominal computed tomography scan confirmed the mass located in the left adrenal area but pointed out multiple other lesions located in the digestive system: on the duodenal papilla $(10 \mathrm{~mm})$ and also colon lesions that could indicate an inflammatory bowel disease. However, the endoscopic biopsy results revealed no malign aspects, only chronic inflammatory infiltrate. In the cardiology clinic, the patient was stabilized by receiving anticoagulant (in prophylactic dose), 
alpha and beta-blocker and antiarrhythmic conversion to sinus rhythm (Figure 3). (amiodarone) treatment, with spontaneous

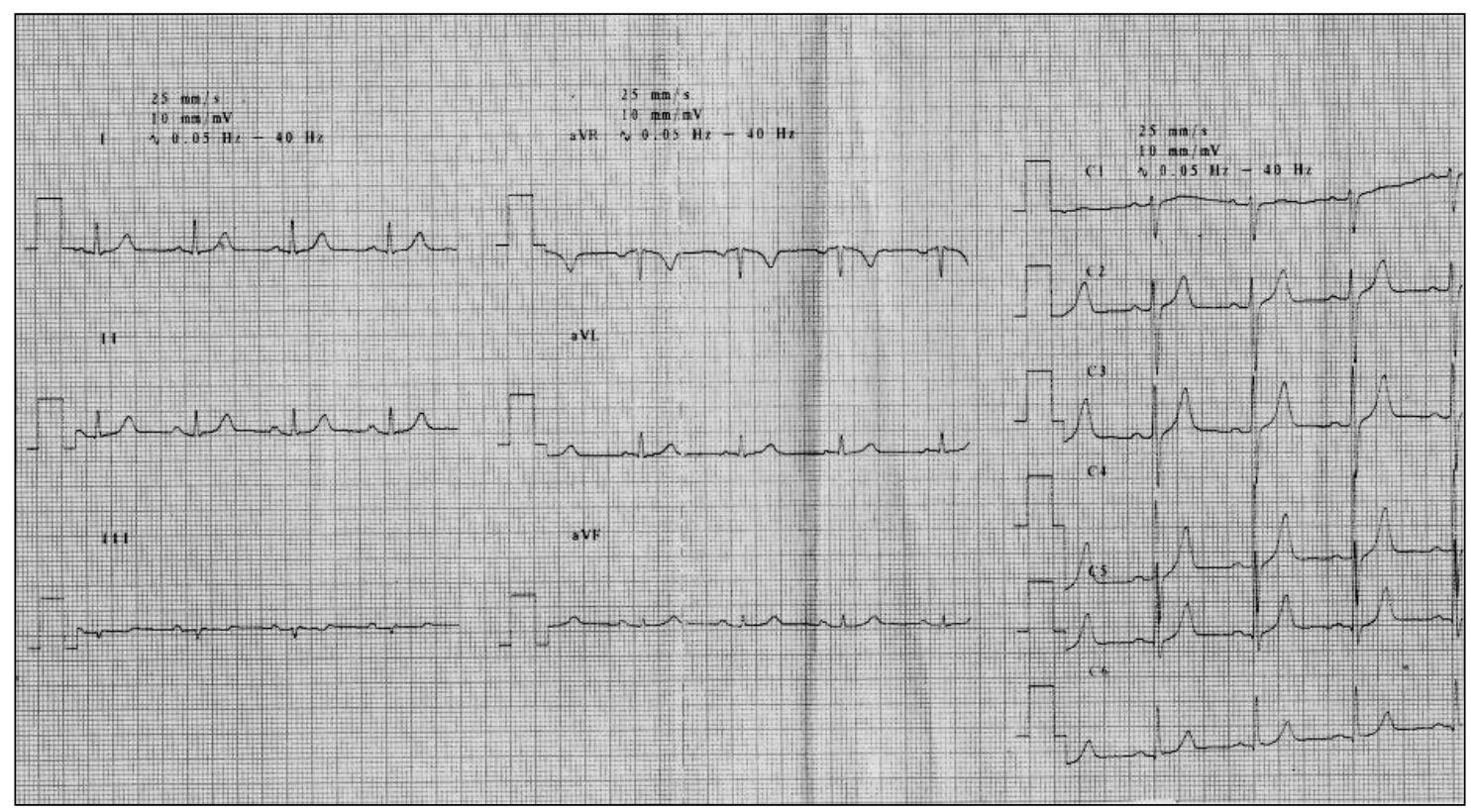

Fig. 3. Sinus rhythm, 75 bpm, intermediate axis, normal wave morphology.

The patient was transferred to the surgery department where the $110 / 110 \mathrm{~mm}$ tumoral mass was removed by abdominal laparotomy (Figure 4).

The histopathological diagnosis confirmed the initial supposition - pheochromocytoma with benign aspect.

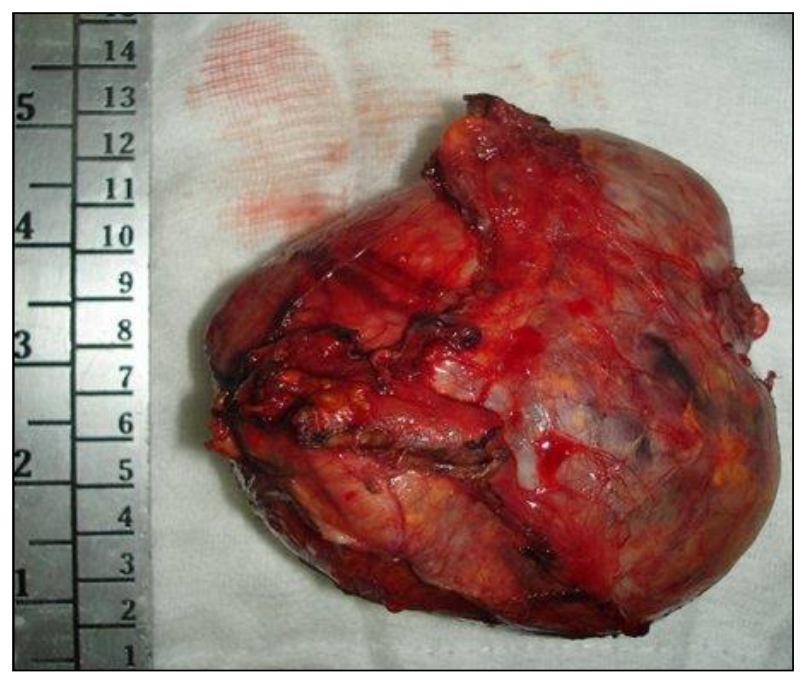

In dynamic, the patient biochemical markers have normalized, the initial values being caused by the adrenergic cardiomyopathy and increased basal metabolism. After the surgery, the evolution was favorable, the patient being scheduled for regular follow-up visits in our department.

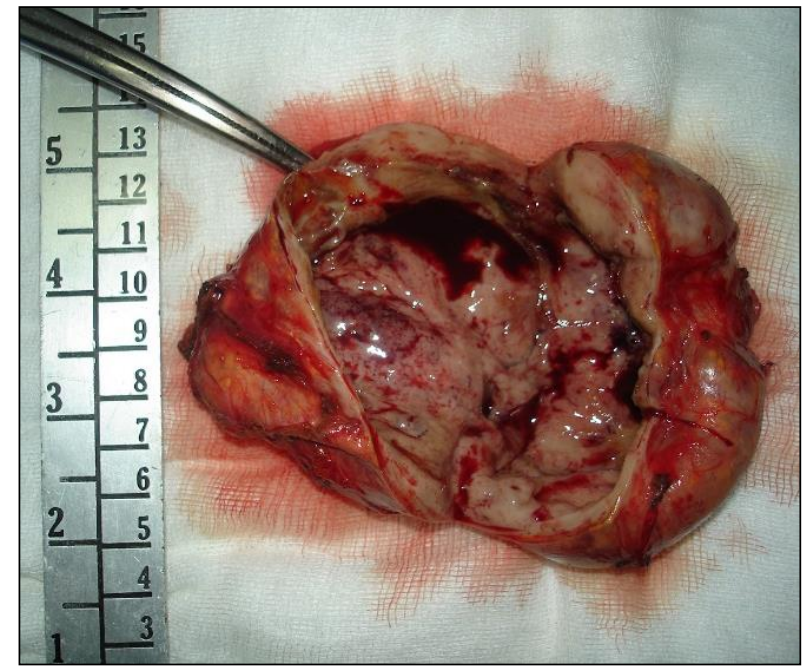

Fig. 4. After surgical excision: 110/110 mm left adrenal tumor - pheochromocytoma 


\section{Discussion}

NF-1 represents a complex disease and the patients have an increased prevalence for both benign and malignant disorders involving neuroectodermal or mesenchymal origin tissues [7]. Despite modern approach and research, the diagnosis is still based on clinical criteria developed by National Institutes of Health Consensus Conference in 1987 [8]. Besides PHEO (which is up to $5 \%$ of cases), gastrointestinal tumors (neuroendocrine tumors of the duodenum, mesenchymal neoplasms or gastrointestinal stromal tumors) occur in up to $20 \%$ of patients [4]. However, very few cases associate at the same time NF1, PHEO and gastrointestinal tumors. In our patient, even though he presented abnormalities at the digestive system, the histopathological exams did not confirm the neoplasia at this level.

Another particular aspect is that we present the case of a 54-year old man with NF-1 and PHEO while this association is predominantly in women around 40 years. Around $20 \%$ of patients are asymptomatic and only $10 \%$ of patients have bilateral adrenal tumors [9].

Neurofibromin (the protein encoded by NF-1 gene) is an important element in the heart development and is expressed in both endothelial and smooth muscle cells of blood vessels [10]. Brannan et al. proved that mice which were homozygous for the Nf1 mutation died prematurely from severe cardiac malformation [11]. About $2 \%$ of patients with NF-1 present cardiovascular malformations, especially pulmonary stenosis [10]. Regarding arterial hypertension, PHEO occurs in $20-50 \%$ of NF-1 hypertensive patients, compared to $0.1 \%$ of all hypertensive subjects [12]. Along with tachyarrhythmia disorder, arterial hypertension was the reason for admission of our patient confirming the acute cardiovascular manifestations that are observed in this category of patients.

A study conducted on 48 consecutive NF1 patients revealed that patients with NF-1 and PHEO presented decreased obesity parameters as compared to NF-1 patients. Moreover, $71 \%$ of them presented higher BP values and a non-dipper profile assessed by ambulatory blood pressure measurement. Left ventricular mass index and intima media thickness were significantly pronounced as well, leading to the conclusion that NF-1 individuals with PHEO had an increased cardiovascular risk [7]. Walther et al. characterized patients with NF-1 and PHEO and showed that mean age of patients was 42 years, $84 \%$ had unilateral adrenal tumors and $11 \%$ presented malignant PHEO [12]. Our patient was evaluated and diagnosed at the age of 54 , probably due to the absence or mild symptoms that did not determine the patient to address earlier to the hospital.

A recent retrospective analysis on PHEO resection concluded that NF-1 patients had significantly smaller adrenal tumors (median tumor dimension, $2.75 \mathrm{~cm}$ vs. $5.9 \mathrm{~cm} ; \mathrm{p}=$ 0.014 ) and less arterial hypertension compared to other subjects treated for PHEO [13]. The results are non-consistent with the literature data probably due to the retrospective character of the study and due to the small number of patients included in the analysis. In our case, the tumor size is not directly associated with the clinical presentation and patient past medical history. However, tumor size as well as open adrenalectomy are independent predictors for intraoperative hemodynamic instability [14].

No specific mutation has been found in patients with NF-1 and PHEO. In a genetic study conducted in 37 subjects, the authors discovered 36 different mutations with no correlation with a certain hot spot [6]. Biochemical and imaging diagnostic and screening tests are not different from those used in sporadic PHEO [15]. Routine screening for PHEO is not recommended in patients with NF-1 because of the low association. However, in hypertensive NF-1 patients, the presence of PHEO should be ruled out.

Children with NF-1 require yearly clinical evaluation and assessment of education and development while adults require lifelong management by a multidisciplinary team [8]. The elective treatment of PHEO is surgical, preferably by laparoscopy, after adequate preparation. The regular strategy includes initial alfa-adrenergic blocker. Beta-blockers should be added after a few days and in the 
presence of an effective alpha-adrenergic blockade since they can worsen the BP profile by inhibiting vasodilatation mediated by beta- 2 adrenergic receptors and thus increasing vasoconstriction [16]. Nowadays, the main choice is represented by cardioselective betablockers.

\section{Conclusions}

There are no general recommendations for the management of patients with NF-1 and PHEO especially when they associate other comorbidities such as arterial hypertension or

Authors declare no conflicts of interest.

\section{References}

1. Pacak K, Linehan WM, Eisenhofer G, Walther MM, Goldstein DS. Recent advances in genetics, diagnosis, localization, and treatment of pheochromocytoma. Ann Intern Med 2001; 134(4):315-329.

2. Rocchietti March M. Type 1 neurofibromatosis and pheochromocytoma: Focus on hypertension. J Neurosci Rural Pract 2012; 3(1):107-108.

3. Zografos GN, Vasiliadis GK, Zagouri F, et al. Pheochromocytoma associated with neurofibromatosis type 1: concepts and current trends. World J Surg Oncol 2010; 8:14.

4. Ozcinar B, Aksakal N, Agcaoglu O, et al. Multiple gastrointestinal stromal tumors and pheochromocytoma in a patient with von Recklinghausen's disease. Int J Surg Case Rep 2013; 4(2):216-218.

5. Lungu B, Baican S, Badarau Z, Lungu Z. Neurofibromatosis Type 1 (von Recklinghausen's disease) in Pregnancy: cesarean delivery outcomes - case report. Arch Clin Cases 2014; 1(2):62-66.

6. Bausch B, Borozdin W, Mautner VF, et al. Germline NF1 mutational spectra and loss-ofheterozygosity analyses in patients with pheochromocytoma and neurofibromatosis type 1. J Clin Endocrinol Metab 2007; 92(7):2784-2792.

7. Zinnamosca L, Petramala L, Cotesta D, et al. Neurofibromatosis type 1 (NF1) and pheochromocytoma: prevalence, clinical and cardiovascular aspects. Arch Dermatol Res 2011; 303(5):317-325.

8. Ferner RE, Huson SM, Thomas N. Guidelines for the diagnosis and management of rhythm disorders as was the case of our patient. We consider the cardiology medical treatment as a milestone for an adequate preparation in order to perform the elective treatment - $\mathrm{PHEO}$ resection.

Even though life expectancy is reduced in patients with such medical conditions, our patient presents a favorable evolution and a proper follow-up is needed.

We highlight the importance of detecting and evaluating cardiovascular signs in all patients with NF-1 since they could unmask a possible secondary cause and disease association.

individuals with neurofibromatosis 1. $\mathrm{J}$ Med Genet 2007; 44(2):81-88.

9. Lew JI, Jacome FJ, Solorzano CC. Neurofibromatosis-associated pheochromocytoma. J Am Coll Surg 2006; 202(3):550-551.

10. Ferner RE. Neurofibromatosis 1 and neurofibromatosis 2: a twenty first century perspective. Lancet Neurol 2007; 6(4):340-351.

11. Brannan $\mathrm{Cl}$, Perkins AS, Vogel KS, et al. Targeted disruption of the neurofibromatosis type-1 gene leads to developmental abnormalities in heart and various neural crestderived tissues. Genes Dev 1994; 8(9):10191029.

12. Walther MM, Herring J, Enquist E, Keiser HR, Linehan WM. von Recklinghausen's disease and pheochromocytomas. J Urol 1999; 162(5):1582-1586.

13. Shinall MC, Solórzano CC. Pheochromocytoma in Neurofibromatosis Type 1: When Should it Be Suspected? Endocr Pract 2014; 20(8):792796.

14. Kiernan CM, Du L, Chen X, et al. Predictors of hemodynamic instability during surgery for pheochromocytoma. Ann Surg Oncol 2014; 21(12):3865-3871.

15. Ollero García-Agulló D, Iriarte Beroiz A, Rojo Alvaro J, Munárriz P, Forga Llenas L. Familial pheochromocytoma associated to neurofibromatosis type 1. Endocrinol Nutr 2013; 60(7):421-422.

16. Satendra M, de Jesus $C$, Bordalo e Sá AL, et al. Reversible catecholamine-induced cardiomyopathy due to pheochromocytoma: case report. Rev Port Cardiol 2014; 33(3):177.e1-6. 